\title{
Health Vigilance for Medical Imaging Diagnostic Optimization: Automated segmentation of COVID-19 lung infection from CT images.
}

\author{
Chala Mohamed ${ }^{3, *}$, Benayad Nsiri ${ }^{1,2}$, Soulaymani Abdelmajid ${ }^{3}$, Mokhtari Abdelghani ${ }^{3}$, and Benaji Brahim ${ }^{1,4,{ }^{*}}$ \\ ${ }^{1}$ Biomedical Engineering Higher School of Technical Education, Mohammed V University Rabat, Morocco \\ ${ }^{2}$ Research Center STIS, M2CS, Higher School of Technical Education of Rabat (ENSET), Mohammed V University Rabat, Morocco \\ ${ }^{3}$ Laboratoire de Génétique et Biométrie University Ibn Tofail Kénitra, Morocco \\ ${ }^{4}$ Group of Bio-pharmaceutical and Toxicology Analysis -Laboratory of Pharmacology and Toxicology-Drugs Sciences Center- \\ Mohammed V University, Rabat, Morocco
}

\begin{abstract}
Covid-19 disease has confronted the world with an unprecedented health crisis, faced with its quick spread, the health system is called upon to increase its vigilance. Se, it is essential to set up a quick and automated diagnosis that can alleviate pressure on health systems. Many techniques used to diagnose the covid-19 disease, including imaging techniques, like computed tomography (CT). In this paper, we present an automatic method for COVID-19 Lung Infection Segmentation from CT Images, that can be integrated into a decision support system for the diagnosis of covid-19 disease. To achieve this goal, we focused to new techniques based on artificial intelligent concept, in particular the uses of deep convolutional neural network, and we are interested in our study to the most popular architecture used in the medical imaging community based on encoder-decoder models. We use an open access data collection for Artificial Intelligence COVID19 CT segmentation or classification as dataset, the proposed model implemented on keras framework in python. A short description of model, training, validation and predictions is given, at the end we compare the result with an existing labeled data. We tested our trained model on new images, we obtained for Area under the ROC Curve the value 0.884 from the prediction result compared with manual expert segmentation. Finally, an overview is given for future works, and use of the proposed model into homogeneous framework in a medical imaging context for clinical purpose.
\end{abstract}

Keywords: Vigilance; Decision Support; Convolutional Neural Network; Image Segmentation; COVID-19

\section{Introduction}

All started in December 2019 [1] in Wuhan city Hubei Province, China, when an epidemic of viral-like pneumonia of unknown etiology has emerged in this city. The virus called Coronavirus 2019 (COVID-19) [2] is an infectious disease caused by a recently discovered coronavirus. The majority of people with COVID-19 will experience only mild to moderate symptoms and will heal without specific treatment.

Given the rapid spread of the WorldWide disease, on March 12, 2020, the World Health Organization (WHO) declared that the covid-19 disease is a pandemic [3].

The world is currently going through the third wave of covid-19, which is characterized by a fast spreading. To date of $03 / 05 / 2021$ [4] more than 153 million Coronavirus Cases covid-19 detected in the world, about a quarter of this was recorded in the USA. In figure 1 we present a tree map chart of countries having nearly $80 \%$ of covid- 19 confirmed cases in the world.

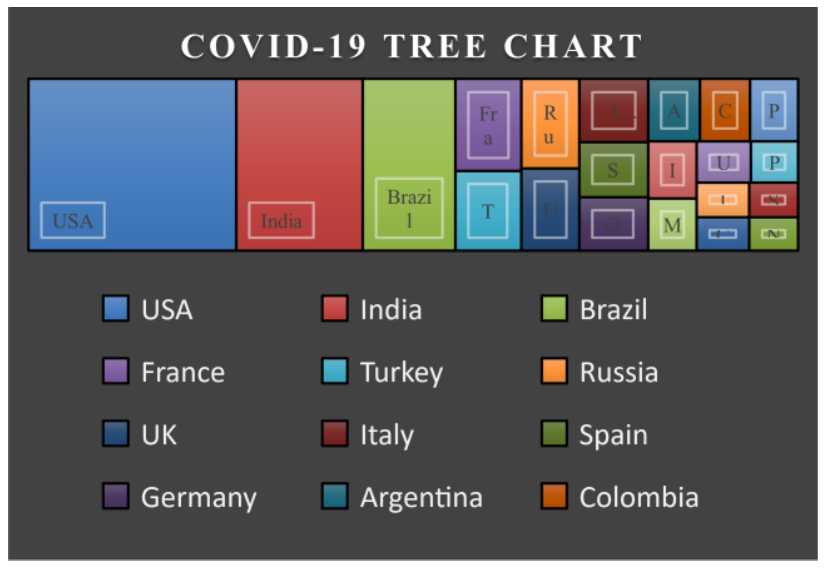

Fig. 1. present a tree map chart of countries having nearly $80 \%$ of covid-19 cases in the world to date $03 / 05 / 2021$.

*Corresponding author: brahim.benaji@um5.ac.ma/mohammed.chala@uit.ac.ma 
The highest cases of COVID-19 is recorded in the United States of America (USA) with over 33 million confirmed cases (May 5, 2021), other countries like India, Brazil, France and Turkey are also influenced by the increase of cases, these five countries sharing more than $51 \%$ of the reported cases in the world.

However, early detection of covid-19 disease is important, not only for rapid treatment initiation, but also for patient isolation and effective public health surveillance. Several techniques and tools used for detection and diagnosing of COVID-19 disease.

We can notice that some virological (RT-PCR) or antigenic tests based on direct samples, it can identify if the person is a carrier of the virus at the time of the test, in which case the caregiver is exposed to a high risk of infection contamination. While other techniques are mainly based on medical imaging including Chest X-ray and Computed Tomography CT [5], [6] which requires less human contact.

In general, techniques used in medical imaging that help to detection and diagnosis of COVID-19 infection are inspired from artificial intelligence (AI). AI is a promising and growing technology for numerous intelligent applications in various areas, we name as an example in literature [7] healthcare, biomedical, fundamental science, language processing, sustainability, engineering, creative industries, autonomous vehicles[8] and more.

In the following, we provide an overview of the application of artificial intelligence in healthcare especially in medical imaging. Many authors cover this subject, Pesapane, F. et al [9] provides some baseline definitions of terms used in AI such as machine learning and deep learning and analyze their integration for radiology purpose. The authors also discuss the use of AI by imaging type (magnetic resonance imaging, Computed tomography, and others), or by subspecialty for radiology or by body part. In [10], the authors gives an overview of state of AI applied to medical cancer imaging and also the progress in its application to lung, brain, breast and prostate tumors. Another uses of IA for brain tumor detection is presented [11], in general, these methods are based on machine learning and deep learning approaches for detection classification or segmentation of tumors from radiology images.

\section{Related works:}

In this section, we present AI-based works for medical imaging processing to covid-19 detection and diagnosis.

\subsection{Convolutional Neural Networks:}

The Convolutional Neural Networks (CNN) is a feed-forward artificial neural network used for visual applications since the $1980^{\prime}$.

In the following, we present some $\mathrm{CNN}$ architectures used in medical imaging analysis to solve classification and segmentation problem.

Fukushima [12], propose a neural network architecture named "neocognitron" for visual pattern recognition tasks, the network is self-learning, it has the ability to recognize stimulus patterns without being changed by a slight distortion of the shape nor by shift in position of the stimulus patterns.

Kaiming et al [13] propose in 2015 a CNN based model named ResNet including residual learning framework for image recognition. The model consists of many blocks for residual learning. Each block can be building as shown in Figure. 2

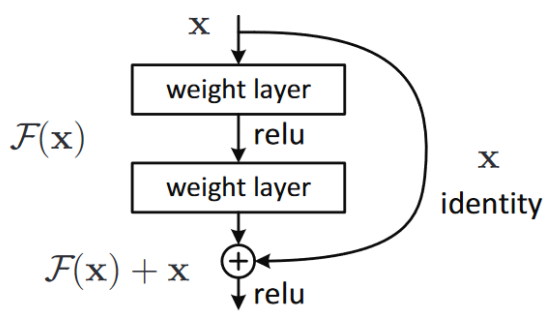

Fig. 2 A building block for residual learning [13].

There are several variants of ResNet models such as ResNet-34, ResNet-50, ResNet-101 and ResNet-152.

Other model called U-Net was presented by Ronneberger et al in [14] for biomedical image segmentation, based on encoderdecoder model, it consists of a series of blocks, each encoder block constitute of convolutional layers followed by a maxpool layer, and for decoder block is deconvolutional layer followed by series of convolutional layers. This model has become one of the most popular architectures used in medical imaging analysis. Figure 3. present the architecture of U-net model.

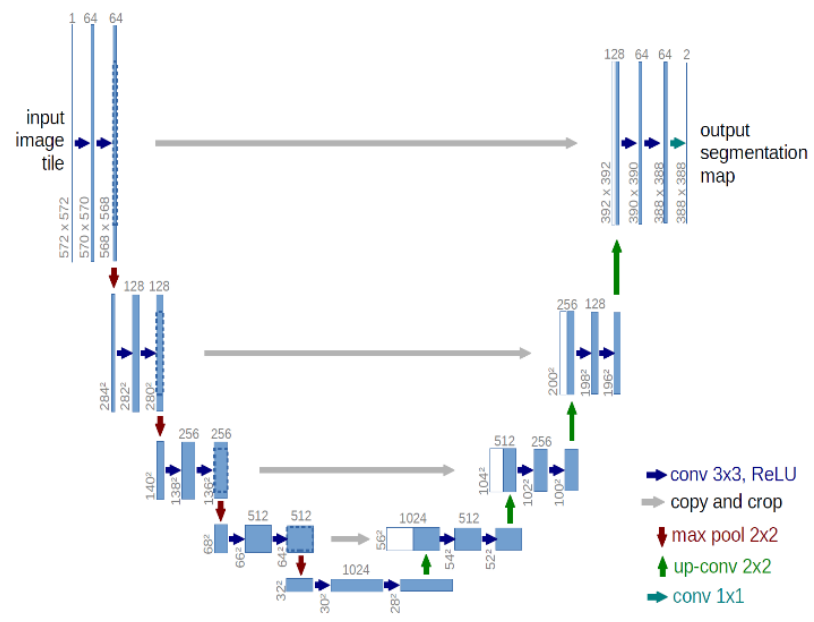

Fig. 3: Show the U-net [14]architecture for biomedical image Segmentation.

Another's interesting models presented by Chala et al in [15], [16], their models based on CNNs architectures, they obtain promising results for automatic segmentation of retinal vessels and optic disc from retinal images.

\subsection{CNN for COVID-19 detection and diagnose:}

Ozturk et al [17] present a novel model based on Convolutional Neural Network (CNN) named DarkCovidNet for detection and classification of COVID-19 from chest X-ray images, the proposed model is able to classify the input chest X-ray images into three classes as COVID-19, Pneumonia, or No-Findings with an accuracy equal to $87.02 \%$. 
Jain et al [18] propose a CNN based method to detect COVID19 cases from X-ray images, the method use tow residual networks to accomplish the work, firstly the use of ResNet50 [13] network to classify the infection into bacterial pneumonia, viral pneumonia or normal, then, the ResNet101[13] network is used in step 2 in order to classify the viral pneumonia into COVID-19 or other and there get a high classification accuracy as $97.77 \%$,

Irmak [19, p. 19] present the use of CNN architecture to detect the COVID-19. The author proposes tow CNN based model, the first model for COVID-19 detection from input chest X-ray image into two classes COVID-19 OR NON COVID-19 98.92\% average accuracy. The second $\mathrm{CNN}$ model is proposed for classification of input chest X-ray image into three classes covid-19 or normal or pneumonia with $98.27 \%$ average accuracy.

Another type of study proposed by Gao et al [20], their method allows to perform a combined classification and lesion segmentation of covid-19 from computed tomography (CT). The proposed framework based on CNN model, for segmentation task the authors use U-net [14] and ResNet-50 with and four residual blocks is utilized for classification task.

Walvekar et al [21] propose an automated image segmentation task based on Deep Leaning techniques, their use the U-net architecture as CNN model for lung segmentation of covid-19 from CT images.

\section{Materials and Method:}

In this section, we present materials and method used for health vigilance and diagnostic optimization through medical imaging.

In order to achieve an artificial intelligent based system for detection and diagnose of covid-19 infection from medical images. We will require datasets. Several open access datasets are publicly available for training deep learning based model, as an example, we cite the dataset described in [22] contain a CT images classified into covid-19 (SARS-CoV-2) virus infection, common pneumonia and normal. Figure 4 show a sample image from this dataset.
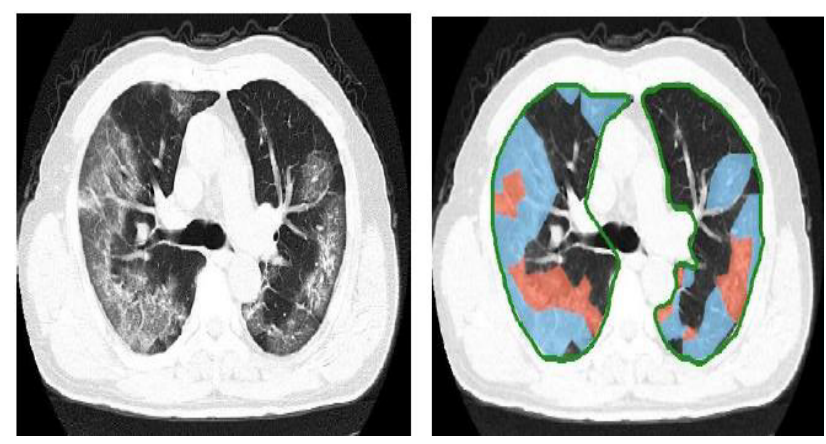

Fig. 4:Sample CT chest image from [19] dataset.

Another CT Images in COVID-19 datasest is presented in [23, p. 19] consists of two unenhanced chest CTs, the first dataset contain 632 patients with COVID-19 infections at initial point of care and the second is a set of 121 CTs from 29 patients with

COVID-19 infections. Figure 5 show a sample image from this dataset.
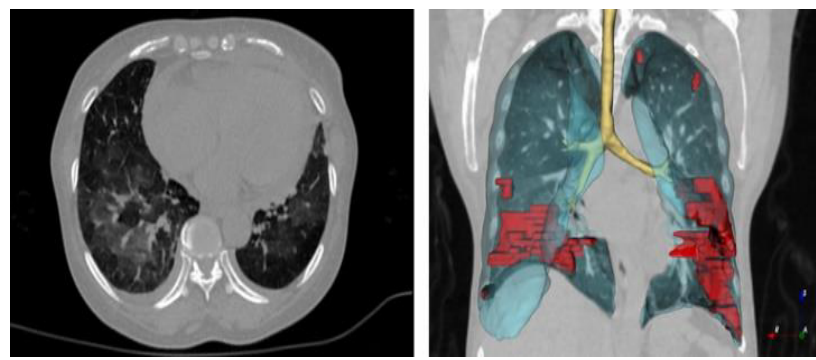

Fig. 5 : Show a sample CT chest image from [20] dataset.

Other public COVID-19 dataset named COVID-19 X-ray image classification [24] is presented in Kaggle competition for COVID-19 cases classification from chest X-ray images. The dataset consists of 15664 with dimension $(512 \times 512)$ chest X-ray images, 15264 images for train set and 400 images for test set. The dataset contains positive and negative classes to indicate the positive and negative COVID-19 cases. Figure 6 show a sample image from this dataset.

Negative
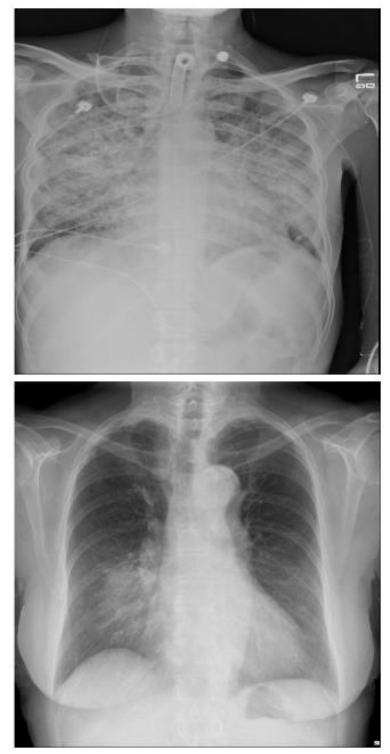

Fig. 6: Show a sample X-Ray chest image from [24] dataset

Once dataset is available, we can choice a CNN model to train in order detect and diagnose the covid-19 lungs infection from medical images like Chest X-ray, CT scan or others.

We present in the Figure.7 our overall proposed system for detection and diagnosis of covid-19 disease.

The proposed system shows on Figure.7 includes four main steps, the first step consists of dataset preparing and preprocessing, the second step consist of building the CNN model with training and testing operations, the CNN proposed model based on U-net [14], this architecture is an encoderdecoder constitute of series of convolutional layer with 
maxpoling layer for encoder step, and series of convolutional layer with deconvolutional layer at end of every level. Once the model is trained and saved, it can be used for prediction from

new chest radiology images. Step three consist of image

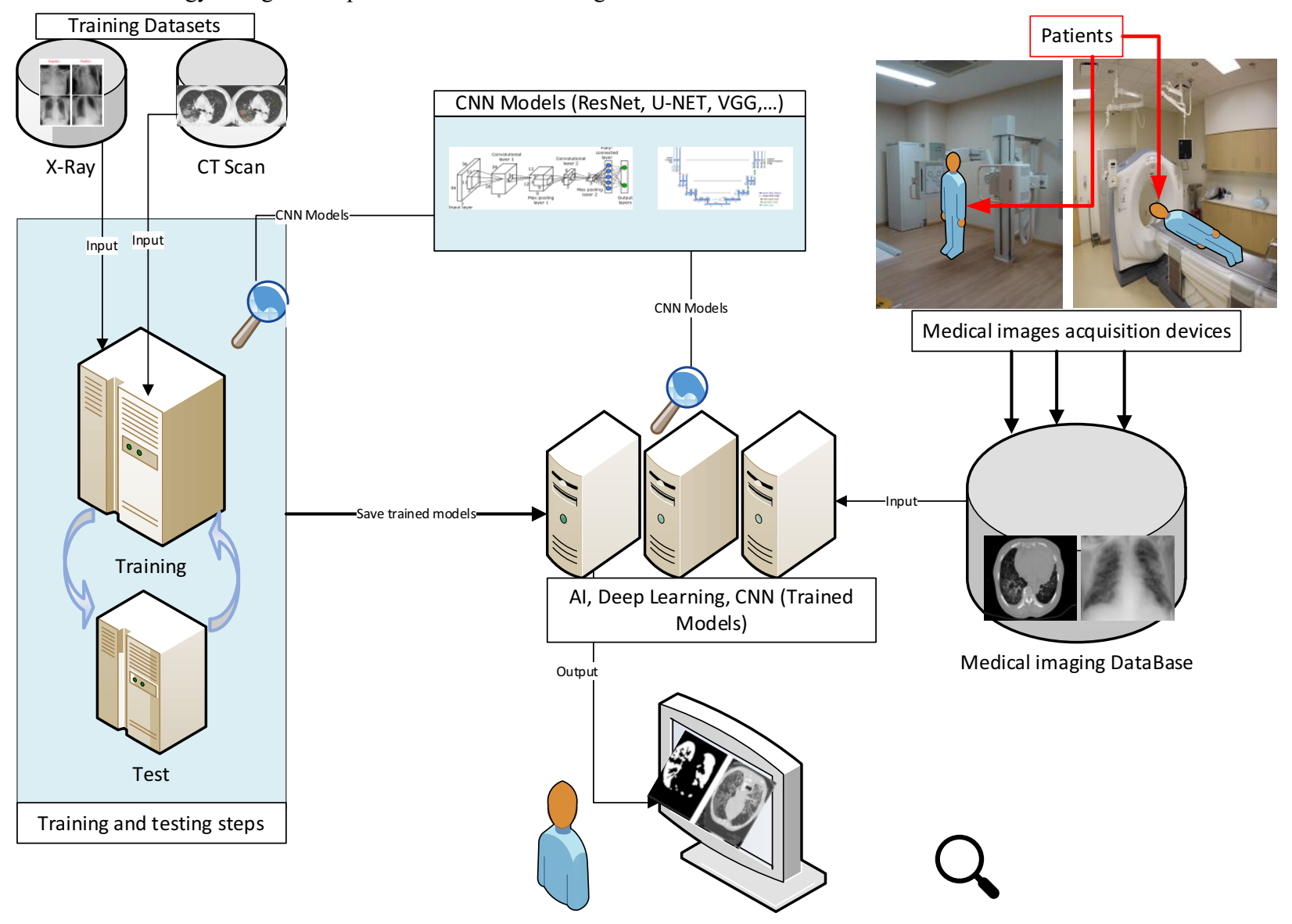

Diagnos decision support systems acquisition from radiological devices for medical imaging analyzes, then the latest step consists the use of new images as input for CNN trained model to predict the covid19 infection.

Fig. 7: The proposed decision support systems for diagnose of covid-19 disease based on artificial intelligent technics

\section{Conclusion:}

In this paper, we present a decision support system including an automatic method based on artificial intelligent to diagnose the covid-19 disease from medical images. The system is a fully automatic framework that allows in parallel to the detection and diagnosis of covid-19 to be used to improve health vigilance by reducing patient contact to a minimum. The system proposed in this paper can be applied to other diseases and used in practice to help health personnel to diagnose other diseases than covid19.

\section{References}

1. Maladie Covid-19 (nouveau coronavirus). Institut Pasteur https://www.pasteur.fr/fr/centre-medical/fichesmaladies/maladie-covid-19-nouveau-coronavirus (2020).

2. World Health Organization. Coronavirus. https://www.who.int/westernpacific/healthtopics/coronavirus (2019).

3. WHO announces COVID-19 outbreak a pandemic. https://www.euro.who.int/en/health-topics/health- emergencies/coronavirus-covid-

19/news/news/2020/3/who-announces-covid-19outbreak-a-pandemic (2020).

4. Coronavirus COVID-19 Virus Pandemic - Worldometer. https://www.worldometers.info/coronavirus/ (2020).

5. Kwee, T. C. \& Kwee, R. M. Chest CT in COVID-19: What the Radiologist Needs to Know. RadioGraphics 40, 1848-1865 (2020).

6. Buzug, T. M. Computed Tomography. in Springer Handbook of Medical Technology (eds. Kramme, R., Hoffmann, K.P. \& Pozos, R. S.) 311-342 (Springer, 2011). doi:10.1007/978-3-540-74658-4 16.

7. Dwivedi, Y. K. et al. Artificial Intelligence (AI): Multidisciplinary perspectives on emerging challenges, opportunities, and agenda for research, practice and policy. Int. J. Inf. Manag. 57, (2021).

8. Ghansiyal, A., Mittal, M. \& Kar, A. K. Information management challenges in autonomous vehicles: A systematic literature review. J. Cases Inf. Technol. 23, 58-77 (2021).

9. Pesapane, F., Codari, M. \& Sardanelli, F. Artificial intelligence in medical imaging: threat or opportunity? Radiologists again at the forefront of innovation in medicine. Eur. Radiol. Exp. 2, 35 (2018). 
10. Bi, W. L. et al. Artificial intelligence in cancer imaging: Clinical challenges and applications. CA Cancer J. Clin. 69, 127-157 (2019).

11. Manogaran, G., Shakeel, P. M., Hassanein, A. S., Malarvizhi Kumar, P. \& Chandra Babu, G. Machine Learning Approach-Based Gamma Distribution for Brain Tumor Detection and Data Sample Imbalance Analysis. IEEE Access 7, 12-19 (2019).

12. Fukushima, K. Neocognitron: A Self-Organizing Neural Network Model for a Mechanism of Pattern Recognition Unaffected by Shift in Position. Biol. Cybern. 36, 193202 (1980).

13. He, K., Zhang, X., Ren, S. \& Sun, J. Deep Residual Learning for Image Recognition. in 2016 IEEE Conference on Computer Vision and Pattern Recognition (CVPR) 770778 (2016). doi:10.1109/CVPR.2016.90.

14. Ronneberger, O., Fischer, P. \& Brox, T. U-Net: Convolutional Networks for Biomedical Image Segmentation. in LNCS vol. 9351 234-241 (2015).

15. Chala, M., Nsiri, B., El yousfi Alaoui, M. H., Soulaymani, A., Mokhtari, A. \& Benaji, B. An automatic retinal vessel segmentation approach based on Convolutional Neural Networks. Expert Syst. Appl. 115459 (2021) doi:10.1016/j.eswa.2021.115459.

16. Chala, M., Nsiri, B., Soulaymani, A., Mokhtari, A. \& Benaji, B. Deep Convolutional Networks based on encoderdecoder architecture for automatic Optic Disc segmentation in retina images. Int. J. Adv. Trends Comput. Sci. Eng. 9, 2078-2084 (2020).

17. Ozturk, T. et al. Automated detection of COVID-19 cases using deep neural networks with X-ray images. Comput. Biol. Med. 121, (2020).

18. Jain, G., Mittal, D., Thakur, D. \& Mittal, M. K. A deep learning approach to detect Covid-19 coronavirus with X-Ray images. Biocybern. Biomed. Eng. 40, 1391-1405 (2020).

19. Irmak, E. Implementation of convolutional neural network approach for COVID-19 disease detection. Physiol. Genomics 52, 590-601 (2020).

20. Gao, K. et al. Dual-branch combination network (DCN): Towards accurate diagnosis and lesion segmentation of COVID-19 using CT images. Med. Image Anal. 67, (2021).

21. Walvekar, S. \& Shinde, S. Efficient Medical Image Segmentation Of COVID-19 Chest CT Images Based on Deep Learning Techniques. in 2021 International Conference on Emerging Smart Computing and Informatics (ESCI) 203-206 (2021). doi:10.1109/ESCI50559.2021.9397043.

22. Zhang, K. et al. Clinically Applicable AI System for Accurate Diagnosis, Quantitative Measurements, and Prognosis of COVID-19 Pneumonia Using Computed Tomography. Cell 181, 1423-1433.e11 (2020).

23. An, P. et al. CT Images in COVID-19. (2020) doi:10.7937/TCIA.2020.GQRY-NC81.

24. COVID-19 X-ray image classification. https://kaggle.com/c/stat946winter2021. 